\title{
Weighted and restricted Deegan-Packel power indices*
}

\author{
Anna Khmelnitskaya ${ }^{\dagger} \quad$ Michela Chessa $^{\ddagger}$
}

September 12, 2020

\begin{abstract}
In the paper we introduce weighted and restricted versions of the Deegan-Packel power index. We show that the classical Deegan-Packel index, which was proposed as an alternative to the Shapley-Shubik index, in fact coincides with the Shapley value of some specific game determined by the set of minimal winning coalitions, and therefore, it has close affinities with the Shapley-Shubik index. We investigate monotonicity properties of the weighted Deegan-Packel index and introduce easy to check conditions under which it is monotonic with respect to the players' weights. An axiomatic characterization of the weighted Deegan-Packel index is provided. The computations done for three real-life examples from realm of politics demonstrate clearly the coincidence of our theoretical predictions with the reality.
\end{abstract}

Keywords: simple games, Deegan-Packel power index, party's weight, communication graph

Mathematics Subject Classification (2000): 91A12, 91A40, 91B14

JEL Classification Number: C71

\section{Introduction}

O2

After parliamentary elections, the situation in which different parties who won a positive number of seats should decide which coalitions to join to form a majority, is quite standard \& on the political podium. In real life it happens quite often that different majorities are $\sum^{\pi}$ feasible and several parties may choose among a number of alternatives. In such cases it is important to have a tool that allows the parties to evaluate properly their actual position $\underset{-\rightarrow}{-1}$ under different opportunities, and therefore, to make a better decision concerning their own 2. choice. On the other hand, the same tool may be used by an external agent intending to predict which coalition is the most likely to form the majority. This problem is usually ${ }_{0}$ modeled from the game theoretical point of view as a simple cooperative game, and a weighted majority game is often applied for this purpose. A solution to such simple game is

${ }^{*}$ The research of Anna Khmelnitskaya was supported by RFBR (Russian Foundation for Basic Research) ${ }_{-}$grant \#16-01-00713 and the French Government scholarship "Metchnikov 2017". Her research was done \% partially during her research stay at Université Côte d'Azur, GREDEG, CNRS, Nice Sophia Antipolis, and $\otimes$ the University of Twente, the hospitality of both universities is highly appreciated.

${ }^{\dagger}$ A.B. Khmelnitskaya, Saint-Petersburg State University, Faculty of Applied Mathematics, Universitetskii prospekt 35, 198504, Petergof, Saint-Petersburg, Russia, e-mail: a.b.khmelnitskaya@utwente.nl

${ }^{\ddagger}$ M. Chessa, Université Côte d’Azur, GREDEG, CNRS, Nice Sophia Antipolis, France, e-mail: michela.chessa@gredeg.cnrs.fr 
called a power index. Several power indices are well known in the literature, for example, the Shapley-Shubik index (cf. Shapley and Shubik [12]), the Banzhaf index (cf. Banzhaf [1]), the Deegan-Packel index (cf. Deegan and Packel [4]), etc. To estimate the power distribution different power indices apply different principles. For example, both Shapley-Shubik and Banzhaf indices take into account the player's participation in all winning coalitions, while the Deegan-Packel index counts only minimal winning coalitions the player belongs to. The latter approach is not only more efficient from computational point of view, but it has been also widely appreciated and accepted by political scientists already for long. For example, in Riker [10] it is shown that there are no circumstances wherein an incentive exists for coalitions of greater than minimal winning size to form and, on the other hand, there are sufficient incentives for such coalitions to form. Also the works of Brams and Fishburn [2] and [3] deserve mentioning in this context. Indeed, an ejection of the superfluous members from a large winning coalition means that the same total amount of value can be divided among the fewer members of its minimal winning subcoalition. Thus, the obtained profit (power) of each of the members, still left in the winning coalition, becomes higher. Moreover, the minimal winning coalitions are also more stable in comparison to the larger ones because all their members have no interest in the loss of any of their partners, which in such a case leads to the loss of winning power of the whole remaining coalition.

However, classical power indices usually evaluate only the ability of parties to form winning coalitions. But for evaluation of the importance of a party in the majority formation not only the party's formal ability to participate in winning coalitions plays a role. Also some other parameters representing asymmetries between the parties such as the party's social impact or social importance reflected in a number of seats the party won during the elections, and also the party's ability to cooperate with the other parties, which may be represented in terms of feasible communication (cooperation) links between the parties, are important. For instance, a simple example of a hypothetical parliament consisting of 99 members and composed by three parties that have correspondingly 49, 49 and 1 seats clearly demonstrates the importance of taking into account the parties' weights when evaluating their power. In fact, while all these three parties play equal roles in the creation of winning coalitions (any two-party coalition in this example is winning) the social evaluation and social respect of the parties are much different. Moreover, any of the two-party coalitions, which includes the last party with only one seat, is no longer winning if one of its members is absent or vote differently from the others, while the coalition formed by the first two parties, each one possessing 49 seats, keeps the winning position even in cases of defections of up to 48 of its members. Unfortunately, the use of the weighted Shapley-Shubik power index, determined by the weighted Shapley value (cf. Shapley [11]) instead of the classical Shapley value (cf. Shapley [11]) used by the Shapley-Shubik index, cannot help to solve the problem. The weighted Shapley-Shubik index indeed takes into account the weights of participants, but as it was noted by Owen [9], the weighted Shapley value and the weighted Shapley-Shubik power index in general and, in particular, the weighted Shapley-Shubik power index in the weighted majority game, may display nonmonotonicity with respect to the players' weights.

In this paper we introduce weighted and restricted versions of the Deegan-Packel power index. We show that the classical Deegan-Packel index, which was proposed by the authors as an alternative to the Shapley-Shubik index ${ }^{1}$, in fact coincides with the Shapley value of

\footnotetext{
${ }^{1}$ Deegan and Packel on page 113 in [4] wrote: "In this paper we propose another power index which, under easily stated assumptions, quantifies an alternate a priori model of power determination."
} 
some specific game determined by the set of minimal winning coalitions, and therefore, it has close affinities with the Shapley-Shubik index. Similarly to the Deegan-Packel index, its weighted version, and also its restricted and weighted restricted versions when restrictions are introduced by means of communication graph, coincide correspondingly with the weighted Shapley value, the Myerson value (cf. Myerson [8]), and the weighted Myerson value (cf. Haeringer [6]) of the same game. We investigate the monotonicity property of the weighted Deegan-Packel index and introduce quite easy to check conditions under which the weighted Deegan-Packel index is monotonic with respect to the players' weights. An axiomatic characterization of the weighted Deegan-Packel index is provided. To illustrate the practical value of the proposed power indices we consider their application for the analysis of three real-life historical examples of parliamentary elections: (i) 1983 Italian Parliament elections after which Bettino Craxi, the leader of the Italian Socialist Party that got only 73 seats in the Lower Chamber from the total amount of 630 , but that had very strong central position, became the Prime Minister; (ii) 2009 Basque Country Parliament elections after which the Basque Nationalist Party winning the largest number of seats finally was not included into the government coalition due to its weak ability to communicate with other political parties; (iii) 2009 Israeli Knesset election after which the Prime Minister, Benjamin Netanyahu, was chosen not from the winning party Kodima, but from the next winning Likud due to its better ability to cooperate with other parties. The computations done show good coincidence of our theoretical predictions with the reality, and thus, clearly demonstrate practical efficiency of applying the proposed power indices.

The structure of the paper is as follows. Preliminaries are given in Section 2. In Section 3 we introduce a weighted Deegan-Packel index, discuss its properties, and provide an axiomatic characterization. Section 4 considers restricted modifications of the DeeganPackel index when not all minimal winning coalitions are feasible. In Section 5 we discuss the application of the introduced indices to analyze three historical examples of parliamentary elections.

\section{Preliminaries}

A cooperative game with transferable utility (TU game) is a pair $(N, v)$, where $N \subset \mathbb{N}$, $N=\{1, \ldots, n\}$, is a finite set of $n$ players and $v: 2^{N} \rightarrow \mathbb{R}$ is a characteristic function with $v(\emptyset)=0$, assigning to every coalition (subset) of players $S \subseteq N$ its worth $v(S)$, which can be freely distributed among the members of $S$. By $\mathcal{G}_{N}$ we denote the set of all TU games with fixed player set $N$. For ease of notation if no ambiguity appears we write $v$ when we refer to a game $(N, v)$. A singleton solution on $\mathcal{G} \subseteq \mathcal{G}_{N}$, usually called a value on $\mathcal{G}$, is a mapping $\xi: \mathcal{G} \rightarrow \mathbb{R}^{N}$ that assigns to every $v \in \mathcal{G}$ a payoff vector $\xi(v) \in \mathbb{R}^{N}$, where $\xi_{i}(v)$ is the payoff to player $i \in N$ in $v$. In the sequel we denote the cardinality of a set $A$ by $|A|$ along with lower case letters like $n=|N|, m=|M|, n_{k}=\left|N_{k}\right|$, etc., and we use standard notation $x(S)=\sum_{i \in S} x_{i}$ for any $x \in \mathbb{R}^{N}$ and $S \subseteq N$.

It is well known (cf. Shapley [11]) that unanimity games $u_{T} \in \mathcal{G}_{N}, T \subseteq N, T \neq \emptyset$, defined as

$$
u_{T}(S)=\left\{\begin{array}{ll}
1, & T \subseteq S, \\
0, & T \nsubseteq S,
\end{array} \quad \text { for all } S \subseteq N,\right.
$$


form a basis in $\mathcal{G}_{N}$, i.e., every $v \in \mathcal{G}_{N}$ can be uniquely presented in the linear form

$$
v=\sum_{T \subseteq N, T \neq \emptyset} \lambda_{T}^{v} u_{T}
$$

where the coefficient $\lambda_{T}^{v}$ is usually referred to as the Harsanyi dividend of coalition $T$ in game $v$ (cf. Harsanyi [5]). One of the most appealing and well known singleton solution concepts is the Shapley value (cf. Shapley [11]) which assigns to a game $v \in \mathcal{G}_{N}$ a payoff vector $S h(v) \in \mathbb{R}^{N}$ given by

$$
S h_{i}(v)=\sum_{\substack{T \subseteq N \\ T \ni i}} \frac{\lambda_{T}^{v}}{t}, \quad \text { for all } i \in N .
$$

Given a vector of positive weights of the players $w \in \mathbb{R}^{N}, w_{i}>0, i \in N$, the weighted Shapley value (cf. Shapley [11]) assigns to a game $v \in \mathcal{G}_{N}$ a payoff vector $S h^{w}(v) \in \mathbb{R}^{N}$ given by

$$
S h_{i}^{w}(v)=\sum_{\substack{T \subseteq N \\ T \ni i}} \lambda_{T}^{v} \frac{w_{i}}{w(T)}, \quad \text { for all } i \in N .
$$

A game $v \in \mathcal{G}_{N}$ is monotonic if for all $S \subseteq T \subseteq N, v(S) \leq v(T)$. Note that every monotonic game is nonnegative, i.e., $v(S) \geq 0$ for all $S \subseteq N$. A monotonic game $v \in$ $\mathcal{G}_{N}$ is simple if it takes only the values 0 and 1 . In a simple game $v$ a coalition $S$ is winning if $v(S)=1$, otherwise $S$ is losing. A coalition $S$ is minimal winning if all its proper subcoalitions $T \varsubsetneqq S$ are losing. It is easy to see that every simple game is uniquely determined by the set of its minimal winning coalitions. For a simple game $v$ the set of all winning coalitions and its subset of all minimal winning coalitions we denote correspondingly by $W(v)$ and $\widehat{W}(v)$. A simple game is proper if for every winning coalition $S \subseteq N$ the complementary coalition $N \backslash S$ is losing. In what follows we concentrate on nontrivial proper simple games, i.e., on proper simple games with the winning grand coalition, and the class of these games we denote by $\mathcal{G}_{N}^{s}$. For any two games $v, v^{\prime} \in \mathcal{G}_{N}^{s}$, a merged game $v \vee v^{\prime} \in \mathcal{G}_{N}^{s}$ is defined as

$$
\left(v \vee v^{\prime}\right)(S)=\left\{\begin{array}{ll}
1, & v(S)=1 \text { or } v^{\prime}(S)=1, \\
0, & \text { otherwise, }
\end{array} \quad \text { for all } S \subseteq N .\right.
$$

Two games $v, v^{\prime} \in \mathcal{G}_{N}^{s}$ are mergeable if for all $S \in \widehat{W}(v)$ and $T \in \widehat{W}\left(v^{\prime}\right), S \nsubseteq T$ and $T \nsubseteq S$, i.e., $\widehat{W}(v) \cap \widehat{W}\left(v^{\prime}\right)=\emptyset$. Observe that for mergeable games $v, v^{\prime} \in \mathcal{G}_{N}^{s}$ it holds that $\widehat{W}\left(v \vee v^{\prime}\right)=\widehat{W}(v) \cup \widehat{W}\left(v^{\prime}\right)$, and therefore,

$$
\left|\widehat{W}\left(v \vee v^{\prime}\right)\right|=|\widehat{W}(v)|+\left|\widehat{W}\left(v^{\prime}\right)\right| .
$$

Given a game $v \in \mathcal{G}_{N}$, a coalition $S \subseteq N$ is a coalition of partners or a $p$-type coalition in $v$ if $v(R \cup T)=v(R)$ for all $T \varsubsetneqq S$ and $R \subseteq N \backslash S$, i.e., every proper subcoalition of a $p$-type coalition is powerless. A player $i \in N$ is a null-player in game $v \in \mathcal{G}_{N}$ if he adds nothing to every coalition not containing him, i.e., $v(S \cup\{i\})=v(S)$ for all $S \subseteq N \backslash\{i\}$. Obviously, in any game, a coalition composed by only null-players is a $p$-type coalition. A player $i \in N$ is a veto-player in game $v \in \mathcal{G}_{N}$ if every coalition not containing him possesses zero worth, i.e., $v(S)=0$ for all $S \subseteq N \backslash\{i\}$. A game $v \in \mathcal{G}_{N}$ is a veto-rich game if it has at least one veto-player. For a simple game $v \in \mathcal{G}_{N}^{s}$ and a winning coalition $S \in W(v)$, a 
player $i \in S$ is pivotal if $S \backslash\{i\}$ is losing, i.e., $S \backslash\{i\} \notin W(v)$. In a veto-rich simple game $v \in \mathcal{G}_{N}^{s}$ a veto-player $i \in N$ is pivotal for every winning coalition $S \subseteq N, S \ni i$. Note that in a simple game $v \in \mathcal{G}_{N}^{s}$, for every $S \in W(v)$, every null-player $i \in S$ is non-pivotal, and for every $S \in \widehat{W}(v)$ every $i \in S$ is pivotal and, therefore, is not a null-player. In a simple game $v \in \mathcal{G}_{N}^{s}$ a player $i \in \cap_{Q \in \widehat{W}(v)} Q$, i.e., a player belonging to every minimal winning coalition, is a veto-player.

Given a tuple $\left[q ; w_{1}, \ldots, w_{n}\right]$ where $q>0$ is an a priori given majority quota and a nonnegative $n$-vector $w=\left(w_{1}, \ldots, w_{n}\right) \in \mathbb{R}_{+}^{N}\left(w_{i} \geq 0, i \in N\right)$ represents the weights (not necessarily normalized to 1 ) of players, a weighted majority game $v^{w}$ on the player set $N$ is a simple game defined as

$$
v^{w}(S)=\left\{\begin{array}{ll}
1, & w(S)>q, \\
0, & \text { otherwise, }
\end{array} \quad \text { for all } S \subseteq N .\right.
$$

When $q \geq \frac{w(N)}{2}$, the corresponding weighted majority game $v^{w}$ is a proper simple game, i.e., $v^{w} \in \mathcal{G}_{N}^{s}$.

A value applied to the subclass of simple games $\mathcal{G}_{N}^{s}$, in particular to simple majority games, is called a power index and each of its components is interpreted as a power assigned to the corresponding player. Several power indices are known from the literature.

The Shapley-Shubik power index (cf. Shapley and Shubik [12]) is defined as the Shapley value of a given simple game and for each player it is determined by all winning coalitions for which the player is critical. For a simple game $v \in \mathcal{G}_{N}^{s}$ the Shapley-Shubik index is given by

$$
S S_{i}(v)=\sum_{\substack{S \notin W(v), S \cup\{i\} \in W(v) \\ S \subseteq N \backslash\{i\}}} \frac{s !(n-s-1) !}{n !}, \quad \text { for all } i \in N .
$$

The Deegan-Packel power index (cf. Deegan and Packel [4]), unlike the Shapley-Shubik index, counts only minimal winning coalitions: the total power is firstly divided equally among minimal winning coalitions and then the power of each of them is divided equally among its members. For a simple game $v \in \mathcal{G}_{N}^{s}$ the Deegan-Packel index is defined as

$$
D P_{i}(v)=\frac{1}{|\widehat{W}(v)|} \sum_{\substack{S \in \widehat{W}(v) \\ S \ni i}} \frac{1}{s}, \quad \text { for all } i \in N \text {. }
$$

A communication structure on $N \subset \mathbb{N}$ is specified by a graph, which is a pair $(N, \Gamma)$, where $N$ is the set of nodes (players) and $\Gamma \subseteq\{\{i, j\} \mid i, j \in N, i \neq j\}$ is the set of links (edges) between two nodes in $N$. For ease of notation and if no ambiguity appears we write $\Gamma$ when we refer to a graph $(N, \Gamma)$. For a graph $\Gamma$ on $N$ and $S \subseteq N$, the subgraph of $\Gamma$ induced by $S$ is the graph $\left.\Gamma\right|_{S}=\{\{i, j\} \in \Gamma \mid i, j \in S\}$ on $S$. In a graph $\Gamma$ a sequence of different nodes $\left(i_{1}, \ldots, i_{r}\right), r \geq 2$, is a path in $\Gamma$ between $i_{1}$ and $i_{r}$ if $\left\{i_{h}, i_{h+1}\right\} \in \Gamma$ for $h=1, \ldots, r-1$. Nodes $i, j \in N, i \neq j$, are connected in graph $\Gamma$ if there exists a path in $\Gamma$ between $i$ and $j . \Gamma$ is connected if any $i, j \in N, i \neq j$, are connected in $\Gamma$. $S \subseteq N$ is connected in $\Gamma$ if $\left.\Gamma\right|_{S}$ is connected. For $S \subseteq N, C^{\Gamma}(S)$ denotes the collection of subsets of $S$ connected in $\Gamma, S / \Gamma$ is the collection of maximal connected subsets, called components, of $S$ in $\Gamma$, and $(S / \Gamma)_{i}$ is the (unique) component of $S$ in $\Gamma$ containing $i \in S$. For ease of notation given a graph $\Gamma$ and a link $\{i, j\} \in \Gamma$, the subgraph $\Gamma \backslash\{\{i, j\}\}$ is denoted by $\Gamma_{-i j}$. 
A pair $(v, \Gamma)$ of a game $v \in \mathcal{G}_{N}$ and a graph $\Gamma$ on $N$ constitutes a game with graph communication structure, for brevity called graph game, on $N$. The set of all graph games with fixed player set $N$ we denote by $\mathcal{G}_{N}^{\Gamma}$. A singleton solution on a set of graph games $\mathcal{G} \subseteq \mathcal{G}_{N}^{\Gamma}$, called a graph game value, or simply value for brevity if no ambiguity appears, on $\mathcal{G}$, is a mapping $\xi: \mathcal{G} \rightarrow \mathbb{R}^{N}$, which assigns to every $(v, \Gamma) \in \mathcal{G}$ a payoff vector $\xi(v, \Gamma) \in \mathbb{R}^{N}$. The Myerson value (cf. Myerson [8]) assigns to a graph game $(v, \Gamma) \in \mathcal{G}_{N}^{\Gamma}$ a payoff vector $\mu(v, \Gamma) \in \mathbb{R}^{N}$ given by

$$
\mu_{i}(v, \Gamma)=S h_{i}\left(v^{\Gamma}\right), \quad \text { for all } i \in N
$$

where

$$
v^{\Gamma}(S)=\sum_{C \in S / \Gamma} v(C), \quad \text { for all } S \subseteq N
$$

Given a vector of positive weights of the players $w \in \mathbb{R}^{N}, w_{i}>0, i \in N$, the weighted Myerson value (cf. Haeringer [6]) assigns to a graph game $(v, \Gamma) \in \mathcal{G}_{N}^{\Gamma}$ a payoff vector $\mu^{w}(v, \Gamma) \in \mathbb{R}^{N}$ given by

$$
\mu_{i}^{w}(v, \Gamma)=S h_{i}^{w}\left(v^{\Gamma}\right), \quad \text { for all } i \in N .
$$

\section{Weighted Deegan-Packel index, properties and axiomatization}

\subsection{Definition and relation to the Shapley value}

Given a simple game $v \in \mathcal{G}_{N}^{s}$ and a vector of positive weights of the players $w \in \mathbb{R}_{++}^{N}$ $\left(w_{i}>0, i \in N\right)$ we assume that unlike the equal distribution of the power of minimal winning coalitions amongst their members as proposed by the original Deegan-Packel index, the power of each minimal winning coalition is distributed proportionally to its members' weights, and we define the weighted Deegan-Packel power index as

$$
D P_{i}^{w}(v)=\frac{1}{|\widehat{W}(v)|} \sum_{\substack{S \in \widehat{W}(v) \\ S \ni i}} \frac{w_{i}}{w(S)}, \quad \text { for all } i \in N \text {. }
$$

Note that the consideration of only positive weights of the players does not restrict the generality. Indeed, in a simple game $v \in \mathcal{G}_{N}^{s}$ every null player $i \in N$ cannot belong to any minimal winning coalition and, therefore, $D P_{i}^{w}(v)=0$ independently of whether the weight $w_{i}=0$ or $w_{i}>0$. From the other hand, the assumption that in a simple game $v \in \mathcal{G}_{N}^{s}$ the weight $w_{i}=0$ of a player $i \in N$, who belongs to at least one minimal winning coalition, is not correct also because it leads to the discrimination of player $i$, since in such a case $D P_{i}^{w}(v)=0$, while as a member of at least one minimal winning coalition $i$ possesses some positive power.

The following example provides good motivation for involving of weighted power indices into analysis of different practical situations, in which the players' weights matter when evaluating their power.

Example 3.1 Consider a hypothetical parliament consisting of 99 members and composed by three parties that have correspondingly 49, 49 and 1 seats. All these three parties play equal roles in the creation of winning coalitions (any two-party coalition in this example is 
winning), but the social evaluation and social respect of the parties differ much. Moreover, any of the two-party coalitions, which includes the last party with only one seat, is no longer winning if one of its members is absent or vote differently from the others, while the coalition formed by the first two parties, each one possessing 49 seats, keeps the winning position even in cases of defections of up to 48 of its members. Simple computations bring the following results:

$$
\begin{aligned}
& \Longrightarrow D P_{i}\left(v^{w}\right)=S h_{i}\left(v^{w}\right)=\frac{1}{3}, \quad i=1,2,3 . \\
& D P_{1}^{w}\left(v^{w}\right)=D P_{2}^{w}\left(v^{w}\right)=\frac{37}{75}=\frac{1221}{2475}, \quad D P_{3}^{w}\left(v^{w}\right)=\frac{1}{75}=\frac{33}{2475}, \\
& S h_{1}^{w}\left(v^{w}\right)=S h_{2}^{w}\left(v^{w}\right)=\frac{1213}{2475}, \quad S h_{3}^{w}\left(v^{w}\right)=\frac{49}{2475},
\end{aligned}
$$

what clearly demonstrates the advantage of use of weighted indices in such situations.

The next theorem reveals close and deep relationship between the Deegan-Packel and the Shapley-Shubik indices and between the weighted Deegan-Packel and the weighted Shapley-Shubik indices correspondingly.

Theorem 3.1 For a simple game $v \in \mathcal{G}_{N}^{s}$, for every $i \in N$ it holds that

$$
D P_{i}(v)=S h_{i}\left(u^{v}\right),
$$

and given a vector of positive weights $w \in \mathbb{R}_{++}^{N}$,

$$
D P_{i}^{w}(v)=S h_{i}^{w}\left(u^{v}\right)
$$

where game $u^{v} \in \mathcal{G}_{N}$ is defined as

$$
u^{v}=\frac{1}{|\widehat{W}(v)|} \sum_{S \in \widehat{W}(v)} u_{S}
$$

Proof. By definition,

$$
D P_{i}(v) \stackrel{(6)}{=} \frac{1}{|\widehat{W}(v)|} \sum_{\substack{S \in \widehat{W}(v) \\ S \ni i}} \frac{1}{s} \stackrel{(2)}{=} \frac{1}{|\widehat{W}(v)|} \sum_{S \in \widehat{W}(v)} S h_{i}\left(u_{S}\right) .
$$

Wherefrom by additivity of the Shapley value and the definition (8) of $u^{v}$ we obtain that

$$
D P_{i}(v)=S h_{i}\left(u^{v}\right) .
$$

The proof for the weighted case is similar and we omit it.

Remark 3.1 For every $v \in \mathcal{G}_{N}^{s}$ it holds that $u^{v}(N)=1$, but in general the game $u^{v}$ is not simple.

Theorem 3.1 implies the following relation between the (weighted) Deegan-Packel and the (weighted) Shapley-Shubik power indices via the (weighted) Shapley value. 
Corollary 3.1 For every simple game $v \in \mathcal{G}_{N}^{s}$, while the (weighted) Shapley-Shubik index by definition is equal to the (weighted) Shapley value of game $v$,

$$
S S_{i}(v)=S h_{i}(v) \quad\left(\text { correspondingly } S S_{i}^{w}(v)=S h_{i}^{w}(v)\right),
$$

the (weighted) Deegan-Packel index is equal to the (weighted) Shapley value of game $u^{v}$,

$$
D P_{i}(v)=S h_{i}\left(u^{v}\right) \quad\left(\text { correspondingly } D P_{i}^{w}(v)=S h_{i}^{w}\left(u^{v}\right)\right) .
$$

The last observation uncovers the deep inner relationship between the Deegan-packel and Shapley-Shubik power indices. Indeed, both indices are given by the Shapley value of two different, but strongly mutually linked games, both of which are uniquely determined via the same set of minimal winning coalitions. While the Shapley-Shubik index is the Shapley value of the given proper simple game, which is uniquely determined by the set of its minimal winning coalitions, however, the nonzero terms in its linear representation (1) via the unanimity basis, in general, may be given by unanimity games determined by any winning coalition and not necessarily only by minimal winning coalition, and therefore, the game, which represents information of all winning coalitions. The Deegan-Packel index is also the Shapley value, but of, in general, not a simple game, which is also uniquely determined by the same set of minimal winning coalitions of the original simple game, but of the game, for which its linear representation via the unanimity basis is given by the mean value of the unanimity games determined by these minimal winning coalitions, and therefore, of the game representing information only over this set of coalitions.

\subsection{Monotonicity with respect to the players' weights}

Unfortunately, similar to the weighted Shapley-Shubik index, the weighted Deegan-Packel index may violate monotonicity with respect to the players' weights. However, while for the weighted Shapley-Shubik index, it is difficult to foresee a priori if in a given simple game the nonmonotonicity effect appears or not, for the weighted Deegan-Packel index there exist easy to check sufficient conditions guaranteeing its monotonic behavior.

Theorem 3.2 Given a simple game $v \in \mathcal{G}_{N}^{s}$ and a vector of positive weights of the players $w \in \mathbb{R}_{++}^{N}$, if $w_{i} \geq w_{j}$ for some $i, j \in N$ and for all $S \in \widehat{W}(v)$ such that $S \ni j, S \not \ni i$, it holds that $(S \backslash\{j\} \cup\{i\}) \in \widehat{W}(v)$, then

$$
D P_{i}^{w}(v) \geq D P_{j}^{w}(v) .
$$

Proof. By definition,

$$
D P_{i}^{w}(v) \stackrel{(7)}{=} \frac{1}{|\widehat{W}(v)|} \sum_{\substack{S \in \widehat{W}(v) \\ S \ni i}} \frac{w_{i}}{w(S)},
$$

which can be equivalently rewritten as

$$
D P_{i}^{w}(v)=\frac{1}{|\widehat{W}(v)|}\left[\sum_{\substack{S \in \widehat{W}(v) \\ S \ni i, S \ni j}} \frac{w_{i}}{w(S)}+\sum_{\substack{S \cup\{i\} \in \widehat{W}(v) \\ S \not \supset i, S \nexists j}} \frac{w_{i}}{w(S)+w_{i}}\right] .
$$

Similarly,

$$
D P_{j}^{w}(v)=\frac{1}{|\widehat{W}(v)|}\left[\sum_{\substack{S \in \widehat{W}(v) \\ S \ni i, S \ni j}} \frac{w_{j}}{w(S)}+\sum_{\substack{S \cup\{j\} \in \widehat{W}(v) \\ S \ngtr i i, S \ngtr j}} \frac{w_{j}}{w(S)+w_{j}}\right] .
$$


Due to the assumption $w_{i} \geq w_{j}$, for all $S \subseteq N \backslash\{i, j\}$, it holds that

$$
\frac{w_{i}}{w(S)} \geq \frac{w_{j}}{w(S)} \quad \text { and } \quad \frac{w_{i}}{w(S)+w_{i}} \geq \frac{w_{j}}{w(S)+w_{j}} .
$$

Further, the assumption that for all $S \in \widehat{W}(v)$ such that $S \ni j, S \not \ni i$, it holds that $(S \backslash\{j\} \cup\{i\}) \in \widehat{W}(v)$, implies that the number of terms in the second sum in (9) is not less then the number of terms in the second sum in (10), and moreover, for each term $\frac{w_{j}}{w(S)+w_{j}}$ in (10) there is the corresponding term $\frac{w_{i}}{w(S)+w_{i}}$ in (9). Wherefrom together with the inequalities (11) we obtain

$$
D P_{i}^{w}(v) \geq D P_{j}^{w}(v)
$$

Furthermore, in a weighted majority game the Deegan-Packel index, similarly to the weighted Deegan-Packel index and unlike the Shapley-Shubik index (see Theorem 3.4 below), may exhibit nonmonotonicity with respect to the players' weights. Fortunately similarly to the just considered situation with the weighted Deegan-Packel index, in this case as well exist easily testable sufficient conditions analogous to those of Theorem 3.2, which guarantee monotonic behavior of the Deegan-Packel index in a weighted majority game.

Theorem 3.3 Given a weighted majority game $v^{w}=\left[q ; w_{1}, \ldots, w_{n}\right] \in \mathcal{G}_{N}^{s}$ with a positive vector of weights of the players $w=\left(w_{1}, \ldots, w_{n}\right) \in \mathbb{R}_{++}^{N}$ and majority quota $q \geq \frac{w(N)}{2}$, if $w_{i} \geq w_{j}$ for some $i, j \in N$ and for all $S \in \widehat{W}\left(v^{w}\right)$, such that $S \ni j, S \not \ngtr i$, it holds that $(S \backslash\{j\} \cup\{i\}) \in \widehat{W}\left(v^{w}\right)$, then

$$
D P_{i}\left(v^{w}\right) \geq D P_{j}\left(v^{w}\right) .
$$

Proof. The proof strategy is similar to the proof of Theorem 3.2. By definition,

$$
D P_{i}\left(v^{w}\right) \stackrel{(6)}{=} \frac{1}{\left|\widehat{W}\left(v^{w}\right)\right|} \sum_{\substack{S \in \widehat{W}\left(v^{w}\right) \\ S \ni i}} \frac{1}{s},
$$

which can be equivalently rewritten as

$$
D P_{i}\left(v^{w}\right)=\frac{1}{\left|\widehat{W}\left(v^{w}\right)\right|}\left[\sum_{\substack{S \in \widehat{W}(v w) \\ S \ni i, S \ni j}} \frac{1}{s}+\sum_{\substack{S \cup\{i\} \in \widehat{W}(v w) \\ S \ngtr i, S \ngtr j}} \frac{1}{s+1}\right] .
$$

Similarly,

$$
\left.D P_{j} v^{w}\right)=\frac{1}{\left|\widehat{W}\left(v^{w}\right)\right|}\left[\sum_{\substack{S \in \widehat{W}(v w) \\ S \ni i, S \ni j}} \frac{1}{s}+\sum_{\substack{S \cup\{j\} \widehat{W}(v w) \\ S \not \supset, S \ngtr j}} \frac{1}{s+1}\right] .
$$

Then, from the definition (4) of a weighted majority game and the assumptions of the theorem, it follows that the number of terms in the second sum in (12) is not less than the number of terms in the second sum in (13). Wherefrom together with equalities (12) - (13) we obtain immediately that

$$
D P_{i}\left(v^{w}\right) \geq D P_{j}\left(v^{w}\right) .
$$


What is indeed remarkable in this context and worth mentioning is that the ShapleyShubik index in a weighted majority game, unlike the weighted Shapley-Shubik index, is always monotonic with respect to the players' weights. However talking about monotonicity, we always keep in mind weak inequalities, which do not exclude equalities. Therefore one should be careful when choosing the Shapley-Shubik and Deegan-Packel indices for investigating the situations, in which it is important to take into account distinctions between the players given in terms of weights, because even in cases when these indices do not violate monotonicity formally they may assign equal power to players with different weights, see for instance Example 3.1.

Theorem 3.4 Given a weighted majority game $v^{w}=\left[q ; w_{1}, \ldots, w_{n}\right] \in \mathcal{G}_{N}^{s}$ with a positive vector of weights of the players $w=\left(w_{1}, \ldots, w_{n}\right) \in \mathbb{R}_{++}^{N}$, if $w_{i} \geq w_{j}$ for some $i, j \in N$, then

$$
S S_{i}\left(v^{w}\right) \geq S S_{j}\left(v^{w}\right)
$$

Proof. By definition,

$$
S S_{i}\left(v^{w}\right) \stackrel{(5)}{=} \sum_{\substack{S \notin W\left(v^{w}\right), S \cup\{i\} \in W\left(v^{w}\right) \\ S \subseteq N \backslash\{i\}}} \frac{s !(n-s-1) !}{n !},
$$

or equivalently,

$S S_{i}\left(v^{w}\right)=\sum_{\substack{S \notin W\left(v^{w}\right), S \cup\{i\} \in W\left(v^{w}\right) \\ S \subseteq N \backslash\{i, j\}}} \frac{s !(n-s-1) !}{n !}+\sum_{\substack{S \cup\{j\} \notin W\left(v^{w}\right), S \cup\{i, j\} \in W\left(v^{w}\right) \\ S \subseteq N \backslash\{i, j\}}} \frac{(s+1) !(n-s) !}{n !}$.

Similarly,

$S S_{j}\left(v^{w}\right)=\sum_{\substack{S \notin W\left(v w^{w}\right), S \cup\{j\} \in W\left(v^{w}\right) \\ S \subseteq N \backslash\{i, j\}}} \frac{s !(n-s-1) !}{n !}+\sum_{\substack{S \cup\{i\} \notin W\left(v^{w}\right), S \cup\{i, j\} \in W\left(v^{w}\right) \\ S \subseteq N \backslash\{i, j\}}} \frac{(s+1) !(n-s) !}{n !}$.

Due to the assumption $w_{i} \geq w_{j}$, from the definition of the weighted majority game (4) it follows that $S \cup\{j\} \in W\left(v^{w}\right)$ implies $S \cup\{i\} \in W\left(v^{w}\right)$ and $S \cup\{i\} \notin W\left(v^{w}\right)$ implies $S \cup\{j\} \notin W\left(v^{w}\right)$. Therefore, the numbers of terms in both sums in (14) are not less than the numbers of terms in both corresponding sums in (15). Whence we immediately obtain that

$$
S S_{i}\left(v^{w}\right) \geq S S_{j}\left(v^{w}\right)
$$

The next example illustrates what was discussed above about the monotonicity issues.

Example 3.2 Consider four weighted majority games $v^{w}, \hat{v}^{w} \bar{v}^{w}$, and $\tilde{v}^{w}$, for which by simple calculations we obtain the following results:

(i) game $v^{w}=[34 ; 30,25,13]$ :

$$
\widehat{W}\left(v^{w}\right)=\{\{1,2\},\{2,3\},\{1,3\}\}
$$




$$
\begin{aligned}
& S S\left(v^{w}\right)=\left(\frac{1}{3}, \frac{1}{3}, \frac{1}{3}\right) \\
& S S^{w}\left(v^{w}\right)=(0.3608,0.3771,0.2621) \\
& D P\left(v^{w}\right)=\left(\frac{1}{3}, \frac{1}{3}, \frac{1}{3}\right) \\
& D P^{w}\left(v^{w}\right)=(0.4144,0.3708,0.2148)
\end{aligned}
$$

(ii) game $\hat{v}^{w}=[10 ; 7,4,3,3,3]$ :

$$
\begin{aligned}
& \widehat{W}\left(\hat{v}^{w}\right)=\{\{1,2\},\{1,3,4\},\{1,4,5\},\{1,3,5\},\{2,3,4,5\}\} \\
& S S\left(\hat{v}^{w}\right)=\left(\frac{27}{60}, \frac{12}{60}, \frac{7}{60}, \frac{7}{60}, \frac{7}{60}\right) \\
& S S^{w}\left(\hat{v}^{w}\right)=(0.4915,0.1654,0.1144,0.1144,0.1144) \\
& D P\left(\hat{v}^{w}\right)=\left(\frac{18}{60}, \frac{9}{60}, \frac{11}{60}, \frac{11}{60}, \frac{11}{60}\right) \\
& D P^{w}\left(\hat{v}^{w}\right)=(0.4503,0.1343,0.1385,0.1385,0.1385)
\end{aligned}
$$

(iii) game $\bar{v}^{w}=[110 ; 79,40,27,27,27]$ :

$$
\begin{aligned}
& \widehat{W}\left(\bar{v}^{w}\right)=\{\{1,2\},\{1,3,4\},\{1,3,5\},\{1,4,5\},\{2,3,4,5\}\} \\
& S S\left(\bar{v}^{w}\right)=\left(\frac{27}{60}, \frac{12}{60}, \frac{7}{60}, \frac{7}{60}, \frac{7}{60}\right) \\
& S S^{w}\left(\bar{v}^{w}\right)=(0.4834,0.1731,0.1145,0.1145,0.1145) \\
& D P\left(\bar{v}^{w}\right)=\left(\frac{18}{60}, \frac{9}{60}, \frac{11}{60}, \frac{11}{60}, \frac{11}{60}\right) \\
& D P^{w}\left(\bar{v}^{w}\right)=(0,4892,0.1667,0.1573,0.1573,0.1573)
\end{aligned}
$$

(iv) game $\tilde{v}^{w}=[50 ; 27,22,17,17,17]$ :

$$
\begin{aligned}
\widehat{W}\left(\tilde{v}^{w}\right)= & \{\{1,2,3\},\{1,2,4\},\{1,2,5\},\{1,3,4\},\{1,3,5\},\{1,4,5\},\{2,3,4\},\{2,3,5\}, \\
& \{2,4,5\},\{3,4,5\}\} \\
S S\left(\tilde{v}^{w}\right)= & \left(\frac{1}{5}, \frac{1}{5}, \frac{1}{5}, \frac{1}{5}, \frac{1}{5}\right) \\
S S^{w}\left(\tilde{v}^{w}\right)= & (0.2090,0.2089,0.1940,0.1940,0.1940) \\
D P\left(\tilde{v}^{w}\right)= & \left(\frac{1}{5}, \frac{1}{5}, \frac{1}{5}, \frac{1}{5}, \frac{1}{5}\right) \\
D P^{w}\left(\tilde{v}^{w}\right)= & (0.2555,0.2179,0.1755,0.1755,0.1755)
\end{aligned}
$$

In games $v^{w}$ and $\tilde{v}^{w}$ the assumptions of Theorems 3.2 and 3.3 are fulfilled and both the Deegan-Packel and weighted Deegan-Packel indices are monotonic with respect to the players' weights. In game $\hat{v}^{w}$ both Deegan-Packel indices manifest nonmonotonic behavior towards the second player in comparison with the last three players, and in game $\bar{v}^{w}$ the Deegan-Packel index violates monotonicity also with respect to the second player in comparison with the last three players, while the weighted Deegan-Packel index is monotonic. But in games $\hat{v}^{w}$ and $\bar{v}^{w}$ the assumptions of Theorems 3.2 and 3.3 are also violated: in 
both these games the sets of minimal winning coalitions coincide and each of three minimal winning coalitions $\{1,3,4\},\{1,4,5\}$, and $\{1,3,5\}$ by replacing any of players 3,4 , or 5 by player 2, keeps being winning but is not anymore a minimal winning. In all games $v^{w}, \hat{v}^{w}$, $\bar{v}^{w}$, and $\tilde{v}^{w}$ the Shapley-Shubik index is monotonic with respect to the players' weights. But the weighted Shapley-Shubik index in game $v^{w}$ demonstrates nonmonotonic behavior with respect to the first and second player, and it exhibits monotonicity in games $\hat{v}^{w}, \bar{v}^{w}$, and $\tilde{v}^{w}$. It is also worth mentioning that even if in games $v^{w}$ and $\tilde{v}^{w}$ both the Shapley-Shubik and Deegan-Packel indices do not violate monotonicity, they both do not distinguish the players according to their weights, by assigning equal power to each one of them.

The table 1 summarizes the behavior of the power indices under scrutiny in four considered simple games on whether they respect monotonicity $(+)$ or not $(-)$.

\begin{tabular}{l|cccc} 
& $v^{w}$ & $\hat{v}^{w}$ & $\bar{v}^{w}$ & $\tilde{v}^{w}$ \\
\hline$S S$ & + & + & + & + \\
$S S^{w}$ & - & + & + & + \\
$D P$ & + & - & - & + \\
$D P^{w}$ & + & - & + & +
\end{tabular}

Table 1: Summary over monotonic behavior of the Shapley-Shubik, weighted ShapleyShubik, Deegan-Packel, and weighted Deegan-Packel power indices.

As a remedy for the complete exclusion of a possible nonmonotonicity issue with respect to the players' weights when determining the power distribution in a weighted majority game by using for this the power distribution mechanism proposed by Deegan and Packel, one may use extended modifications of the Deegan-Packel and weighted Deegan-Packel indices, which differently from the Deegan-Packel and weighted Deegan-Packel indices take into account not only minimal winning but all winning coalitions.

For a simple game $v \in \mathcal{G}_{N}^{s}$, the extended Deegan-Packel index is defined as

$$
\widetilde{D P}_{i}(v)=\frac{1}{|W(v)|} \sum_{\substack{S \in W(v) \\ S \ni i}} \frac{1}{s}, \quad \text { for all } i \in N \text {. }
$$

For a simple game $v \in \mathcal{G}_{N}^{s}$ and a vector of positive weights of the players $w \in \mathbb{R}_{++}^{N}$, the weighted extended Deegan-Packel index is defined as

$$
\widetilde{D P}_{i}^{w}(v)=\frac{1}{|W(v)|} \sum_{\substack{S \in W(v) \\ S \ni i}} \frac{w_{i}}{w(S)}, \quad \text { for all } i \in N .
$$

Theorem 3.5 Given a weighted majority game $v^{w}=\left[q ; w_{1}, \ldots, w_{n}\right] \in \mathcal{G}_{N}^{s}$ with a positive vector of weights of the players $w=\left(w_{1}, \ldots, w_{n}\right) \in \mathbb{R}_{++}^{N}$, if $w_{i} \geq w_{j}$ for some $i, j \in N$, then

$$
\widetilde{D P}_{i}\left(v^{w}\right) \geq \widetilde{D P}_{j}\left(v^{w}\right) \quad \text { and } \quad \widetilde{D P}_{i}^{w}\left(v^{w}\right) \geq \widetilde{D P}_{j}^{w}\left(v^{w}\right)
$$

The proofs of both inequalities in Theorem 3.5 can be easily obtained by simple adaptation of the proofs of Theorems 3.2 and 3.3, and therefore we omit them.

However, when applying the extended versions of the Deegan-Packel indices it is necessary to take into account that these indices differ principally from the original ones because they violate the basic postulate of Deegan and Packel [4] stating that only the minimal winning coalitions matter in evaluating the power of different participants. 


\subsection{Axiomatization}

The weighted Deegan-Packel power index on $\mathcal{G}_{N}^{s}$ can be characterized via next five axioms:

A power index $\xi$ is efficient $(E)$ on $\mathcal{G}_{N}^{s}$ if for all $v \in \mathcal{G}_{N}^{s}$ it holds that $\sum_{i \in N} \xi_{i}(v)=1$.

A power index $\xi$ meets null-player property $(N P)$ on $\mathcal{G}_{N}^{s}$, if for all $v \in \mathcal{G}_{N}^{s}$ and every null-player $i \in N$ in $v$ it holds that $\xi_{i}(v)=0$.

A power index $\xi$ on $\mathcal{G}_{N}^{s}$ is strictly positive $(S P)$ if for all $v \in \mathcal{G}_{N}^{s}$ and every $i \in N$, which is not a null-player in $v$, it holds that $\xi_{i}(v)>0$.

A power index $\xi$ meets mergeability $(M)$ on $\mathcal{G}_{N}^{s}$ if for any two mergeable games $v, v^{\prime} \in \mathcal{G}_{N}^{s}$ it holds that

$$
\xi\left(v \vee v^{\prime}\right)=\frac{1}{\left|\widehat{W}\left(v \vee v^{\prime}\right)\right|}\left[|\widehat{W}(v)| \xi(v)+\left|\widehat{W}\left(v^{\prime}\right)\right| \xi\left(v^{\prime}\right)\right]
$$

A power index $\xi$ meets partnership $(P)$ on $\mathcal{G}_{N}^{s}$ if for any $v \in \mathcal{G}_{N}^{s}$ and every $p$-type coalition $S \subseteq N$ in $v$ it holds that

$$
\xi_{i}(v)=\xi_{S}(v) \xi_{i}\left(u_{S}\right), \quad \text { for all } i \in S
$$

The intuition content and rationale behind these axioms is consistent with the desired properties of a power index. Efficiency, null-player, and strict positivity are standard axioms used in various axiomatizations, in particular for the Shapley value. Mergeability, used in Deegan and Packel [4] for the characterization of the Deegan-Packel index, states that the power in a merged game of two mergeable games is a weighted mean of the powers of the component games with the weights provided by the numbers of minimal winning coalitions in each component game. Partnership, introduced in Kalai and Samet [7] for the characterization of the weighted Shapley values, requires that if the proper subcoalitions of a coalition are powerless, then it should not make any difference whether players of the coalition receive their individual payoffs in the game, or they altogether receive their group payoff in the game and then later determine their individual payoffs. The original requirement of the Kalai and Samet Partnership axiom is reformulated a bit here to make the axiom acceptable for application on the class of simple games $\mathcal{G}_{N}^{s}$. Note that for the KalaiSamet axiomatization of the weighted Shapley values the current modification is equivalent to their original Partnership axiom due to the linearity of the weighted Shapley values.

The next lemma reveals the structure of $p$-type coalitions for games in $\mathcal{G}_{N}^{s}$ which is needed for the proof of the axiomatization result below.

Lemma 3.1 Given a game $v \in \mathcal{G}_{N}^{s}$, a coalition $S \subseteq N$ is of $p$-type in $v$ if and only if it is of one of the following types:

(i) every $i \in S$ is a null-player in $v$;

(ii) every $i \in S$ is a not null-player in $v$ and there is $Q \in \widehat{W}(v)$ such that $Q \supseteq S$; moreover if $|\widehat{W}(v)|>1$, one of the two options also should hold:

(ii.a) $S \cap Q^{\prime}=\emptyset$ for all $Q^{\prime} \in \widehat{W}(v), Q^{\prime} \neq Q$;

(ii.b) $S \subseteq Q^{\prime}$ for all $Q^{\prime} \in \widehat{W}(v)$. 
Proof. It is not difficult to see that in all three cases $(i)$, (ii.a), and (ii.b) a coalition $S$ is of $p$-type in $v$ :

$(i)$ : From the definition of a null-player it follows immediately that $v(R \cup T)=v(R)$ for all $T \varsubsetneqq S$ and $R \subseteq N \backslash S$.

(ii): In this case for every $T \varsubsetneqq S$ and $R \subseteq N \backslash S$ it also holds true that $v(R \cup T)=v(R)$. Indeed, if $v(R)=1$, then the validity of the latter equality follows from the monotonicity of game $v$. If $v(R)=0$, the equality $v(R \cup T)=1$ is impossible because if it is true, there should exist $Z \in \widehat{W}(v)$ such that

$$
R \cup T \supseteq Z \text {, but } R \nsupseteq Z \text {. }
$$

However, if $Z=Q,(18)$ is impossible because $Q \supseteq S \supsetneqq T, R \subseteq N \backslash S$, and therefore, $R \cup T \nsupseteq Q$. But if $|\widehat{W}(v)|>1$ and $Z=Q^{\prime}$ for $Q^{\prime} \in \widehat{W}(v), Q^{\prime} \neq Q,(18)$ is also impossible. Indeed, in case of (ii.a) it holds that $S \cap Q^{\prime}=\emptyset$ for all $Q^{\prime} \in \widehat{W}(v), Q^{\prime} \neq Q$, and therefore, $R \cup T \supseteq Q^{\prime}$ can be only if $Q^{\prime} \subseteq R$, which is impossible because of the assumption $v(R)=0$. In alternative case of (ii.b), every minimal winning coalition contains $S$, i.e., every $i \in S$ is a veto-player, whence together with $T \varsubsetneqq S$ and $R \subseteq N \backslash S$ it follows immediately that $v(R \cup T)=v(R)=0$. Hence, if $v(R)=0$, then $v(R \cup T)=0$ as well.

It is left to show that a $p$-type in $v$ coalition $S$ containing at least one not null-player is a coalition of type (ii.a) or (ii.b). First we show that all players $i \in S$ are not null-players as well. We prove that by contradiction. Let $i \in S$ be not a null-player and besides assume that there is at least one null-player in $S$. Then there is a coalition $Z \subset N \backslash\{i\}$ such that $v(Z)=0$ and $v(Z \cup\{i\})=1$. Since player $i$ is pivotal for $Z \cup\{i\} \in W(v)$ there is $Q \in \widehat{W}(v)$ such $Q \subseteq Z \cup\{i\}, Q \ni i$, and moreover, $Q \cap S \varsubsetneqq S$ because minimal winning coalition cannot contain null-players. Let $T=Q \cap S \varsubsetneqq S$ and $R=Q \cap(N \backslash S)$. Then $v(R)=0$ since game $v$ is proper and monotonic, but $v(R \cup T)=v(Q)=1$, which contradicts to $S$ being of $p$-type.

We show now that for any $p$-type in $v$ coalition $S$ composed by not null-players there is $Q \in \widehat{W}(v)$ such that $Q \supseteq S$, and if $|\widehat{W}(v)|>1$, then either $S \cap Q^{\prime}=\emptyset$ for all $Q^{\prime} \in \widehat{W}(v)$, $Q^{\prime} \neq Q$, or $S \subseteq Q^{\prime}$ for all $Q^{\prime} \in \widehat{W}(v)$. We consider two cases $S \in W(v)$ and $S \notin W(v)$.

1). Let $S \in W(v)$, i.e., $v(S)=1$. We show that this is possible only if $S$ is a unique minimal winning coalition, i.e. $S \in \widehat{W}(v)$ and $|\widehat{W}(v)|=1$. Assume that $S \notin \widehat{W}(v)$. Then there exists $T \in \widehat{W}(v)$ such that $T \varsubsetneqq S . v(N \backslash S)=0$ because $S \in W(v)$ and $v$ is a proper simple game. Take $R \subseteq N \backslash S$. By monotonicity of $v, v(R)=0$, but since $T \in \widehat{W}(v)$, $v(R \cup T)=1$, which contradicts to $S$ being of $p$-type, and therefore, $S \in \widehat{W}(v)$.

Next we show that a minimal winning coalition $S \in \widehat{W}(v)$ can be of $p$-type only if being a unique one, i.e., if $|\widehat{W}(v)|=1$. Indeed, if there is $Q \in \widehat{W}(v), Q \neq S$, then $Q \cap S \neq \emptyset$ and $v(Q \backslash S)=0$ because game $v$ is proper and monotonic. Consider $T=Q \cap S \varsubsetneqq S$ and $R=Q \backslash S \subset N \backslash S$. Then, $v(R)=0$, but $v(R \cup T)=v(Q)=1$, again contradiction with $S$ being of $p$-type. Wherefrom it follows that $|\widehat{W}(v)|=1$.

2 ). Let $S \notin W(v)$, i.e., $v(S)=0$. It was just proved above that for every not null-player $i \in S$ there is $Q \in \widehat{W}(v)$ such that $Q \cap S \ni i$ and that all $i \in S$ are not null-players. So, to prove the statement it is enough to show that for $p$-type coalition $S$ composed by only not null-players it is impossible that two different minimal winning coalitions have nonempty intersections with $S$, or all minimal winning coalitions contain $S$, i.e., $S$ is composed by only veto-players. 
Indeed, assume that there are $Q_{1}, Q_{2} \in \widehat{W}(v), Q_{1} \neq Q_{2}$, such that $Q_{j} \cap S \neq \emptyset, j=1,2$, and that there is a player $i \in S$, such that $i \in Q_{1}$, but $i \notin Q_{2}$. Consider $T=Q_{2} \cap S \varsubsetneqq S$ and $R=Q_{2} \backslash S \subseteq N \backslash S$. Then, $v(R)=0$, but $v(R \cup T)=v\left(Q_{2}\right)=1$, which contradicts to $S$ being of $p$-type.

Corollary 3.2 A p-type coalition $S \subseteq N$ in a game $v \in \mathcal{G}_{N}^{s}$ is winning only if $v$ has a single minimal winning coalition, which, in turn, coincides with $S$, i.e., $|\widehat{W}(v)|=1$ and $S \in \widehat{W}(v)$. This implies that unanimity games are the only games in $\mathcal{G}_{N}^{s}$ that have $p$-type winning coalitions, and, moreover, every unanimity game $u_{S}, S \subseteq N$, has the only $p$-type minimal winning coalition, which is $S$.

Theorem 3.6 A power index $\xi$ on $\mathcal{G}_{N}^{s}$ meets efficiency, null-player property, strict positivity, mergeability, and partnership if and only if there exists a vector of strictly positive weights $w \in \mathbb{R}_{++}^{N}$ such that $\xi=D P^{w}$.

Proof. I. First we show that the weighted Deegan-Packel index meets the axioms E, NP, $\mathrm{SP}, \mathrm{M}$ and P. Assume that a proper simple game $v \in \mathcal{G}_{N}^{s}$ and a vector of weights $w \in \mathbb{R}_{++}^{N}$ $w=\left(w_{i}\right)_{i \in N}, w_{i}>0, i \in N$, are given.

$(\mathrm{E})$ :

$$
\sum_{i \in N} D P_{i}^{w}(v) \stackrel{(7)}{=} \sum_{i \in N} \frac{1}{|\widehat{W}(v)|} \sum_{\substack{S \in \widehat{W}(v) \\ S \ni i}} \frac{w_{i}}{w(S)}=\frac{1}{|\widehat{W}(v)|} \sum_{S \in \widehat{W}(v)} \sum_{i \in S} \frac{w_{i}}{w(S)}=1 .
$$

(NP): The null-player property follows immediately from the fact that a null-player in $v$ does not belong to any minimal winning coalition.

(SP): The strict positivity is a direct corollary of the assumption of strict positivity of the players' weights: $w_{i}>0, i \in N$.

$(\mathrm{M})$ : Let games $v, v^{\prime} \in \mathcal{G}_{N}^{s}$ be mergeable. To confirm the mergeability of the weighted Deegan-Packel index we need to verify the equality (16) for $\xi=D P^{w}$. Indeed, since for mergeable games $v$ and $v^{\prime}$ it holds that $\widehat{W}(v) \cap \widehat{W}\left(v^{\prime}\right)=\emptyset$ and $\widehat{W}\left(v \vee v^{\prime}\right)=\widehat{W}(v) \cup \widehat{W}\left(v^{\prime}\right)$, we obtain that for all $i \in N$,

$$
\begin{aligned}
D P_{i}^{w}\left(v \vee v^{\prime}\right) & \stackrel{(7)}{=} \frac{1}{\left|\widehat{W}\left(v \vee v^{\prime}\right)\right|} \sum_{\substack{S \in \widehat{W}\left(v \vee v^{\prime}\right) \\
S \ni i}} \frac{w_{i}}{w(S)} \\
& =\frac{1}{\left|\widehat{W}\left(v \vee v^{\prime}\right)\right|}\left[\sum_{\substack{S \in \widehat{W}(v) \\
S \ni i}} \frac{w_{i}}{w(S)}+\sum_{\substack{S \in \widehat{W}\left(v^{\prime}\right) \\
S \ni i}} \frac{w_{i}}{w(S)}\right] \\
& \stackrel{(7)}{=} \frac{1}{\left|\widehat{W}\left(v \vee v^{\prime}\right)\right|}\left[|\widehat{W}(v)| D P_{i}^{w}(v)+\left|\widehat{W}\left(v^{\prime}\right)\right| D P_{i}^{w}\left(v^{\prime}\right)\right] .
\end{aligned}
$$

(P): Let coalition $S \subseteq N$ be of $p$-type in $v$. To prove the partnership property of the weighted Deegan-Packel index we need to show that equality (17) with $\xi=D P^{w}$ holds true, or which is the same to prove that for all $i \in S$,

$$
\frac{1}{|\widehat{W}(v)|} \sum_{\substack{T \in \widehat{W}(v) \\ T \ni i}} \frac{w_{i}}{w(T)}=\left[\sum_{j \in S}\left(\frac{1}{|\widehat{W}(v)|} \sum_{\substack{T \in \widehat{W}(v) \\ T \ni j}} \frac{w_{j}}{w(T)}\right)\right] \frac{w_{i}}{w(S)}
$$


Due to Lemma 3.1 a $p$-type coalition in $v$ can be only of one of the three types $(i),(i i . a)$, or $($ ii.b):

If $(i)$, then all $i \in S$ are null-players in $v$ and the null-player property of the weighted Deegan-Packel index implies $D P_{i}^{w}(v)=D P_{S}^{w}(v)=0$, wherefrom equality (19) obviously follows.

If (ii.a), then equality (19) reduces to

$$
\frac{w_{i}}{w(S)}=\left[\sum_{j \in S} \frac{w_{j}}{w(S)}\right] \frac{w_{i}}{w(S)}
$$

which obviously confirms the validity of equality (19) as well.

If (ii.b), $S \subseteq T$ for all $T \in \widehat{W}(v)$, and therefore, for all $i \in S$ it holds that $\{T \in \widehat{W}(v) \mid$ $T \ni i\}=\{T \in \widehat{W}(v)\}$. Hence, in this case equality (19) transforms into

$$
\sum_{T \in \widehat{W}(v)} \frac{w_{i}}{w(T)}=\left[\sum_{j \in S} \sum_{T \in \widehat{W}(v)} \frac{w_{j}}{w(T)}\right] \frac{w_{i}}{w(S)} .
$$

But then it is not difficult to see that the right side of the latter equality easily reduces to the left one:

$$
\left[\sum_{j \in S} \sum_{T \in \widehat{W}(v)} \frac{w_{j}}{w(T)}\right] \frac{w_{i}}{w(S)}=\sum_{T \in \widehat{W}(v)} \frac{\sum_{j \in S} w_{j}}{w(T)} \frac{w_{i}}{w(S)}=\sum_{T \in \widehat{W}(v)} \frac{w_{i}}{w(T)}
$$

what also confirms equality (19).

II. Second, to complete the proof of the theorem, we show that every power index $\xi$ on $\mathcal{G}_{N}^{s}$ satisfying axioms E, NP, SP, M, and P, coincides with the weighted Deegan-Packel index with some particular vector of positive weights $w \in \mathbb{R}_{++}^{N}$.

First we define the vector of weights $w$ as

$$
w_{i}=\xi_{i}\left(u_{N}\right), \quad \text { for all } i \in N .
$$

Since there are no null-players in the unanimity game $u_{N} \in \mathcal{G}_{N}^{s}$, the strict positivity of the weighted Deegan-Packel index implies $w \in \mathbb{R}_{++}^{N}$.

Consider arbitrary game $v \in \mathcal{G}_{N}^{s}$. Obviously, for any distinct $Q, Q^{\prime} \in \widehat{W}(v)$, the unanimity games $u_{Q}, u_{Q^{\prime}} \in \mathcal{G}_{N}^{s}$ are mergeable. Moreover, for any pairwise mergeable games $v, v^{\prime}, v " \in \mathcal{G}_{N}^{s}$, games $v \vee v^{\prime}$ and $v^{\prime}$ are mergeable, and $\widehat{W}\left(v \vee v^{\prime}\right)=\widehat{W}(v) \cup \widehat{W}\left(v^{\prime}\right)$. Whence it straightforwardly follows that

$$
v=\vee_{Q \in \widehat{W}(v)} u_{Q} .
$$

Due to the latter equality and since both indices $\xi$ and $D P^{w}$ satisfy mergeability, the successive application of mergeability (16) together with equality (3) leads to the equalities

$$
\xi_{i}(v)=\frac{\sum_{Q \in \widehat{W}(v)} \xi_{i}\left(u_{Q}\right)}{|\widehat{W}(v)|}
$$

and

$$
D P_{i}^{w}(v)=\frac{\sum_{Q \in \widehat{W}(v)} D P_{i}^{w}\left(u_{Q}\right)}{|\widehat{W}(v)|}
$$


Hence, to prove coincidence of the $\xi$ and $D P^{w}$ indices it is enough to show that they coincide on the unanimity games.

So, given a unanimity game $u_{S} \in \mathcal{G}_{N}^{s}, S \subseteq N, S \neq \emptyset$, we need to show that for all $i \in N$,

$$
\xi_{i}\left(u_{S}\right)=D P_{i}^{w}\left(u_{S}\right)
$$

First note that every $i \in N \backslash S$ is a null-player in $u_{S}$, and the null-player property of both $\xi$ and $D P^{w}$ implies that for all $i \in N \backslash S$,

$$
\xi_{i}\left(u_{S}\right)=D P^{w}\left(u_{S}\right)=0 .
$$

Then, from Lemma 3.1 it follows that $S$ is a $p$-type coalition in $u_{S}$. Since $\xi$ satisfies partnership (17), it holds that for any game $v \in \mathcal{G}_{N}^{s}$,

$$
\xi_{i}(v)=\xi_{S}(v) \xi_{i}\left(u_{S}\right), \quad \text { for all } i \in S .
$$

Wherefrom together with the definition of weights $w_{i}, i \in N$, and the definition of the $D P^{w}$-index it follows that for all $i \in S$,

$$
\xi_{i}\left(u_{S}\right)=\frac{\xi_{i}\left(u_{N}\right)}{\xi_{S}\left(u_{N}\right)} \stackrel{(20)}{=} \frac{w_{i}}{w(S)} \stackrel{(7)}{=} D P_{i}^{w}\left(u_{S}\right)
$$

\section{Restricted and weighted restricted Deegan-Packel indices}

It might happen that in a given simple game $v \in \mathcal{G}_{N}^{s}$ not all winning coalitions are feasible. In such a case instead of the Deegan-Packel and weighted Deegan-Packel indices we may consider the restricted Deegan-Packel and weighted restricted Deegan-Packel indices. In what follows the set of all feasible winning coalitions of game $v \in \mathcal{G}_{N}^{s}$ we denote by $W^{F}(v)$ and by $\widehat{W^{F}}(v)$ the set of all feasible minimal winning coalitions.

For a simple game $v \in \mathcal{G}_{N}^{s}$, the restricted Deegan-Packel index is defined as

$$
D P_{i}^{r}(v)=\frac{1}{\left|\widehat{W^{F}}(v)\right|} \sum_{\substack{S \in \widehat{W^{F}}(v) \\ S \ni i}} \frac{1}{s}, \quad \text { for all } i \in N .
$$

For a simple game $v \in \mathcal{G}_{N}^{s}$ and a vector of positive weights of the players $w \in \mathbb{R}_{++}^{N}$, the weighted restricted Deegan-Packel index is defined as

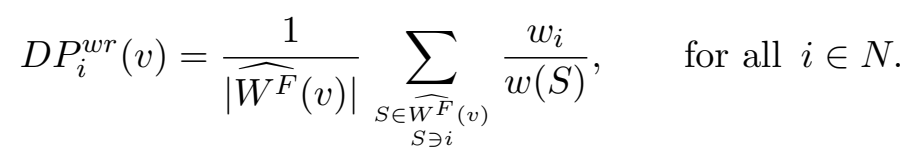

When restrictions on cooperation are given by an undirected connected graph $\Gamma$ on $N$, instead of only a simple game $v \in \mathcal{G}_{N}^{s}$ we have a tuple of a simple game $v$ together with a graph communication structure $\Gamma$, which determines a graph game $(v, \Gamma)$. Following Myerson (1977), we assume that cooperation is possible only among connected players and consider a (Myerson) restricted game $v^{\Gamma}$ on $N$ defined as

$$
v^{\Gamma}(S)=\sum_{C \in S / \Gamma} v(C), \quad \text { for all } S \subseteq N .
$$


Observe that since all simple games in $\mathcal{G}_{N}^{s}$ are monotonic and proper, for every $v \in \mathcal{G}_{N}^{s}$, the restricted game $v^{\Gamma} \in \mathcal{G}_{N}^{s}$ as well and, moreover, every minimal winning coalition in $v^{\Gamma}$ is connected in $\Gamma$ and winning in $v$. From where it easily follows that in this case the set of feasible minimal winning coalitions in $(v, \Gamma)$ coincides with the set of minimal winning coalitions in $v^{\Gamma}$, i.e., $\widehat{W^{F}}(v, \Gamma)=\widehat{W}\left(v^{\Gamma}\right)$.

Similar to Theorem 3.1 revealing the relation between the (weighted) Deegan-Packel and the (weighted) Shapley-Shubik indices via the (weighted) Shapley value, the next theorem uncovers the similar relation between the (weighted) restricted Deegan-Packel index in case, when restrictions on cooperation are introduced by undirected communication graph, and the (weighted) Myerson index via the (weighted) Myerson value, and the (weighted) Shapley-Shubik index of the restricted game via the (weighted) Shapley value.

Theorem 4.1 For a graph game $(v, \Gamma) \in \mathcal{G}_{N}^{\Gamma}$ given by a simple game $v \in \mathcal{G}_{N}^{s}$, for every $i \in N$ it holds that

$$
D P_{i}^{r}(v, \Gamma)=\mu_{i}\left(\bar{u}^{v}, \Gamma\right)=S h_{i}\left(\left(\bar{u}^{v}\right)^{\Gamma}\right),
$$

and given a vector of positive weights $w \in \mathbb{R}_{++}^{N}$,

$$
D P_{i}^{w r}(v, \Gamma)=\mu_{i}^{w}\left(\bar{u}^{v}, \Gamma\right)=S h_{i}^{w}\left(\left(\bar{u}^{v}\right)^{\Gamma}\right),
$$

where game $\bar{u}^{v} \in \mathcal{G}_{N}$ is defined as

$$
u^{v}=\frac{1}{\left|\widehat{W}\left(v^{\Gamma}\right)\right|} \sum_{S \in \widehat{W}\left(v^{\Gamma}\right)} u_{S} .
$$

Moreover, when restrictions on cooperation are introduced by an undirected graph, for the restricted Deegan-Packel and weighted restricted Deegan-Packel indices, similar to the Deegan-Packel and weighted Deegan-Packel indices, there exist easy to check sufficient conditions guaranteeing their monotonic behavior with respect to the players' weights. In fact these conditions are practically the same, as for the Deegan-Packel and weighted DeeganPackel indices given by Theorems 3.2 and 3.3, with the only replacement of game $v$ by the restricted game $v^{\Gamma}$ in the adapted statements of the theorems.

Theorem 4.2 For a graph game $(v, \Gamma) \in \mathcal{G}_{N}^{\Gamma}$ given by a simple game $v \in \mathcal{G}_{N}^{s}$ and a vector of positive weights of the players $w \in \mathbb{R}_{++}^{N^{N}}$, if $w_{i} \geq w_{j}$ for some $i, j \in N$ and for all $S \in \widehat{W}\left(v^{\Gamma}\right)$ such that $S \ni j, S \not \supset i$, it holds that $(S \backslash\{j\} \cup\{i\}) \in \widehat{W}\left(v^{\Gamma}\right)$, then

$$
D P_{i}^{w r}(v, \Gamma) \geq D P_{j}^{w r}(v, \Gamma) .
$$

Theorem 4.3 Given a weighted majority game $v^{w}=\left[q ; w_{1}, \ldots, w_{n}\right] \in \mathcal{G}_{N}^{s}$ with a positive vector of weights of the players $w=\left(w_{1}, \ldots, w_{n}\right) \in \mathbb{R}_{++}^{N}$ and majority quota $q \geq \frac{w(N)}{2}$, and a communication graph $\Gamma$ on $N$, if $w_{i} \geq w_{j}$ for some $i, j \in N$ and for all $S \in \widehat{W}\left(\left(v^{w}\right)^{\Gamma}\right)$, such that $S \ni j, S \not \ni i$, it holds that $(S \backslash\{j\} \cup\{i\}) \in \widehat{W}\left(\left(v^{w}\right)^{\Gamma}\right)$, then

$$
D P_{i}^{r}\left(v^{w}, \Gamma\right) \geq D P_{j}^{r}\left(v^{w}, \Gamma\right) .
$$

The proof strategies of Theorems 4.1-4.3 are similar to that in corresponding Theorems 3.1-3.3, and therefore these proofs are omitted. 


\section{$5 \quad$ Historical examples}

To illustrate a practical value of the introduced power indices we consider their application for the analysis of three historical examples of parliamentary elections.

\subsection{Italian parliament elections 1983}

An impressive historical example when a small but central party that got not too many seats in a parliament but finally became the most important in the sense that a prime minister was chosen from it can be found in the Italian parliament elections of 1983 . The official data on the distribution of seats in the Chamber of Deputies are:

Democrazia Cristiana (DC) 225 seats;

Partito Comunista Italiano (PCI) 198 seats;

Partito Socialista Italiano (PSI) 73 seats;

Movimiento Sociale It. (MSI-DN) 42 seats;

Partito Repubblicano Italiano (PRI)

29 seats;

Partito Socialista Dem. It. (PSDI)

23 seats;

Partito Liberale Italiano (PLI)

16 seats;

Partito Radicale (PR)

Democrazia Proletaria (DP)

11 seats;

Sudtiroler Volkspartei (SVP)

7 seats;

Liga Veneta (LV)

Partito Sardo d'Azione (PSA)

3 seats;

UV-UVP - Dem.Pop.

and possible links between the parties ${ }^{2}$ are depicted in Figure 1.

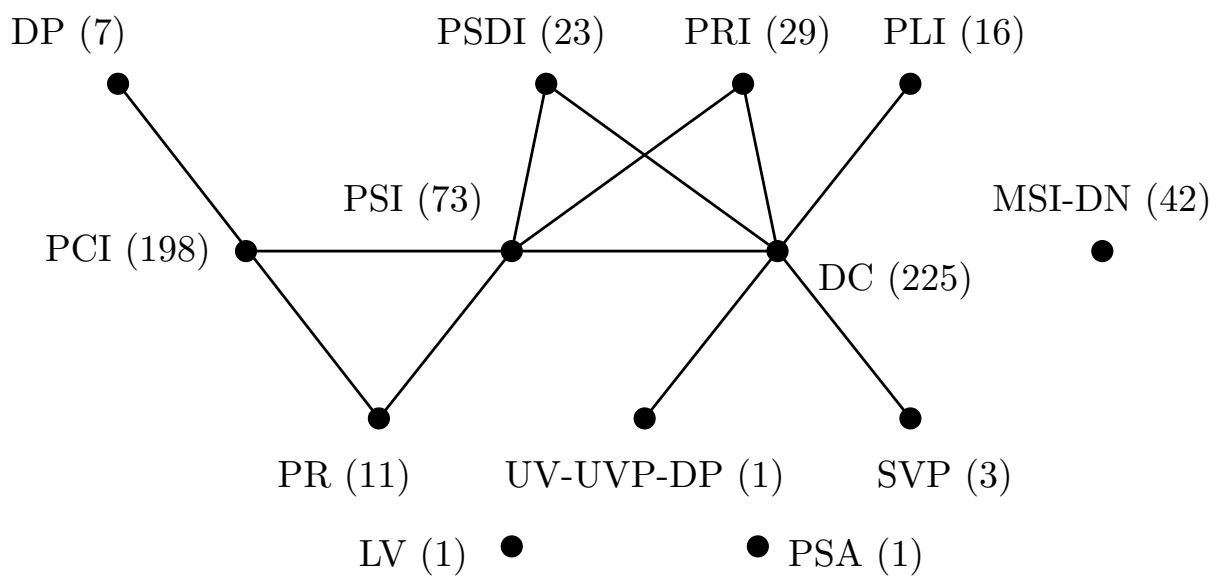

Figure 1: Italian parliament elections 1983, network structure.

Following this elections the prime minister, Bettino Craxi, was chosen from Italian Socialist Party (PSI) that after elections got only 73 seats from the total amount of 630 in the Chamber of Deputies. But PSI possessed very strong bargaining power being between two

\footnotetext{
${ }^{2}$ The information about possible links between the parties is provided by Vito Fragnelli, Italian game theorist working in the field of power indices and their applications, who is well familiar with the historical relations between Italian political parties.
} 
main parties - Christian Democrats (DC) that got 225 seats and Italian Communist Party (PCI) that got 198 seats. Indeed, the traditional ruling coalition, known as Pentapartito (five parties), was DC + PLI + PSI + PRI + PSDI $(225+16+73+29+23=366)$. But there was another possible winning coalition composed by PCI + PSI + DP + PR + PSDI + PRI $(198+73+7+11+23+29=341)$. The latter coalition never formed before on the country government level but it happened to be formed on the levels of local governments. This fact provided PSI with very strong bargaining power and finally the prime-minister Bettino Craxi, member of PSI, was chosen.

To analyze the situation formally, consider the corresponding weighted majority game, which is a standard tool for analysis of voting situations. The total number of seats is 630 , so, the ruling coalition should contain at least 316 members. The minimal winning coalitions in the graph-restricted game are DC + PCI + PSI, DC + PSI + PRI, DC + PSI + PSDI, PCI + PSI + PRI + PSDI and PCI + PSI + PRI + PR + DP. Italian Socialist Party appears in all of them, whereas the two most voted parties, Christian Democrats and Communists appear only in three each one, and moreover, their union is not feasible due to the ideological incompatibilities. Consequently, PSI appears to be a veto-player in the restricted gamethe formation of ruling coalitions is impossible ignoring PSI. The computation results on power distribution according to the studied in the paper power indices, the Deegan-Packel, weighted Deegan-Packel, restricted Deegan-Packel, and weighted restricted Deegan-Packel indices, are presented in Table 2.

\begin{tabular}{c|c|c|c|c|c}
\hline Party & Seats & $D P$ & $D P^{w}$ & $D P^{r}$ & $D P^{r w}$ \\
\hline \hline Democrazia Cristiana (DC) & 225 & 0.109 & 0.363 & 0.194 & 0.465 \\
\hline Partito Comunista Italiano (PCI) & 198 & 0.098 & 0.307 & 0.075 & 0.206 \\
\hline Partito Socialista Italiano (PSI) & 73 & 0.122 & 0.127 & 0.269 & 0.227 \\
\hline Movimiento Sociale It. (MSI-DN) & 42 & 0.098 & 0.068 & 0 & 0 \\
\hline Partito Repubblicano Italiano (PRI) & 29 & 0.089 & 0.047 & 0.131 & 0.045 \\
\hline Partito Socialista Dem. It. (PSDI) & 23 & 0.071 & 0.031 & 0.097 & 0.024 \\
\hline Partito Liberale Italiano (PLI) & 16 & 0.093 & 0.026 & 0.083 & 0.017 \\
\hline Partito Radicale (PR) & 11 & 0.069 & 0.015 & 0.075 & 0.011 \\
\hline Democrazia Proletaria (DP) & 7 & 0.070 & 0.010 & 0.033 & 0.004 \\
\hline Sudtiroler Volkspartei (SVP) & 3 & 0.056 & 0.004 & 0.042 & 0.002 \\
\hline Liga Veneta (LV) & 1 & 0.042 & 0.001 & 0 & 0 \\
\hline Partito Sardo d'Azione (PSA) & 1 & 0.042 & 0.001 & 0 & 0 \\
\hline UV-UVP - Dem.Pop. & 1 & 0.042 & 0.001 & 0 & 0 \\
\hline
\end{tabular}

Table 2: Italian parliament elections 1983, power distribution according to the DeeganPackel type power indices.

One can see immediately that the power of PSI, both according to the classical and weighted Deegan-Packel indices, increases significantly when the underlying communication structure is taken into account. In particular, PSI is the party with the highest power value when applying the restricted Deegan-Packel index. The value of its power slightly decreases under using the weighted restricted Deegan-Packel index when also the weights given by the seats shares are taken into account. 


\subsection{Basque Country parliament elections 2009}

The Basque Country (Euskadi) is one of 17 autonomous communities of Spain. Its parliament elections 2009 provide an example when the party that won the elections but being not able to create the majority itself finally was not included into the government due to the weak cooperation ability. The Basque Country parliament contains 75 members and after the elections in March 2009 it was composed by 7 parties with the distribution of seats as follows:

$\begin{array}{lr}\text { Basque Nationalist Party (PNV) } & 30 \text { seats; } \\ \text { Euskadi's Socialist Party (PSE) } & 25 \text { seats; } \\ \text { Popular Party (PP) } & 13 \text { seats; } \\ \text { ARALAR } & 4 \text { seats; } \\ \text { Euzko Alkartasuna (EA) } & 1 \text { seat; } \\ \text { Ezker Batua-Berdeak (EB-B) } & 1 \text { seat; } \\ \text { Union, Progress and Democracy (UPD) } & 1 \text { seat, }\end{array}$ Plausible links between the parties, which take into account the historical relations among parties in Spain, are depicted in Figure 2.

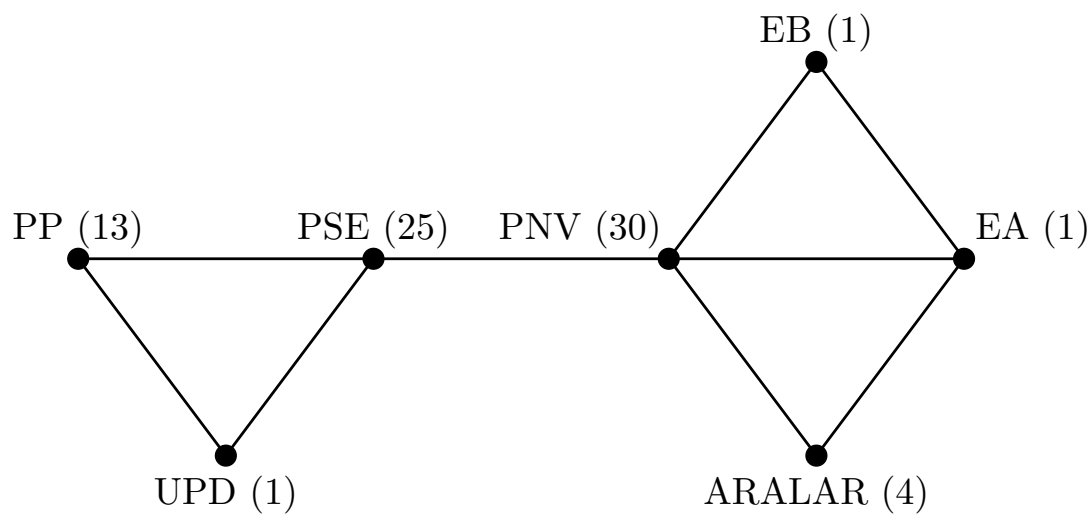

Figure 2: Basque Country parliament elections 2009, network structure.

With the total number of seats of 75 , a majority coalition contains at least 38 members. Notice the lack of a direct relation between the right PP and the nationalist PNV parties. Whereas PSE and PNV had formed government coalitions in the Basque Country in the past, such a coalition was never formed in case of both PP and PNV parties being present together. Moreover, the strong rejection of the nationalism in the discourse of PP is an additional reason to consider the combination of $\mathrm{PP}$ and $\mathrm{PNV}$ to be impossible because of its (predictably) high electoral cost for the PP in the rest of Spain. As the result, in 2009 PSE preferred to form a ruling coalition together with PP and UPD, without including the most voted nationalist PNV. Table 3 presents the computation results of the Deegan-Packel type indices considered in the paper. 


\begin{tabular}{c|c|c|c|c|c}
\hline Party & Seats & $D P$ & $D P^{w}$ & $D P^{r}$ & $D P^{r w}$ \\
\hline \hline Basque Nationalist Party (PNV) & 30 & $1 / 3$ & 0.414 & $1 / 4$ & 0.273 \\
\hline Euskadi's Socialist Party (PSE) & 25 & $1 / 3$ & 0.371 & $1 / 2$ & 0.556 \\
\hline Popular Party (PP) & 13 & $1 / 3$ & 0.215 & $1 / 4$ & 0.171 \\
\hline ARALAR & 4 & 0 & 0 & 0 & 0 \\
\hline Euzko Alkartasuna (EA) & 1 & 0 & 0 & 0 & 0 \\
\hline Ezker Batua-Berdeak (EB-B) & 1 & 0 & 0 & 0 & 0 \\
\hline Union, Progress and Democracy (UPD) & 1 & 0 & 0 & 0 & 0 \\
\hline
\end{tabular}

Table 3: Basque Country parliament elections 2009, power distribution according to the Deegan-Packel type power indices.

Note that the Deegan-Packel index assigns equal power to the three main parties, as they symmetrically belong to three two-player minimal winning coalitions. However, both the restricted Deegan-Packel and weighted restricted Deegan-Packel indices clearly highlight the highest power of PSE, because of its crucial position in the communication structure.

\subsection{Israeli 18th Knesset elections 2009}

After the Israeli Knesset elections 2009, similarly to the Basque Country parliament elections 2009 discussed in the previous example, it happened that the most voted party Kadima was not included into the ruling coalition created by Likud who received in Knesset less seats.

Following the elections the 18th Knesset of 120 members was composed as:

$\begin{array}{lr}\text { Kadima } & 28 \text { seats; } \\ \text { Likud } & 27 \text { seats; } \\ \text { Yisrael Beiteinu } & 15 \text { seats; } \\ \text { Avoda } & 13 \text { seats; } \\ \text { Shas } & 11 \text { seats; } \\ \text { United Torah Judaism + National Union } & 5+4=9 \text { seats; } \\ \text { Ta'al } & 4 \text { seats. } \\ \text { Hadash } & 4 \text { seats. } \\ \text { Balad } & 3 \text { seats. } \\ \text { The Jewish Home } & 3 \text { seats. } \\ \text { Meretz } & 3 \text { seats. }\end{array}$

Plausible links between the parties based on the historical relations between the Israeli parties are depicted in Figure 3. Given the closeness of the United Torah Judaism and National Union positions and for the sake of simplicity, we put these two parties together. 


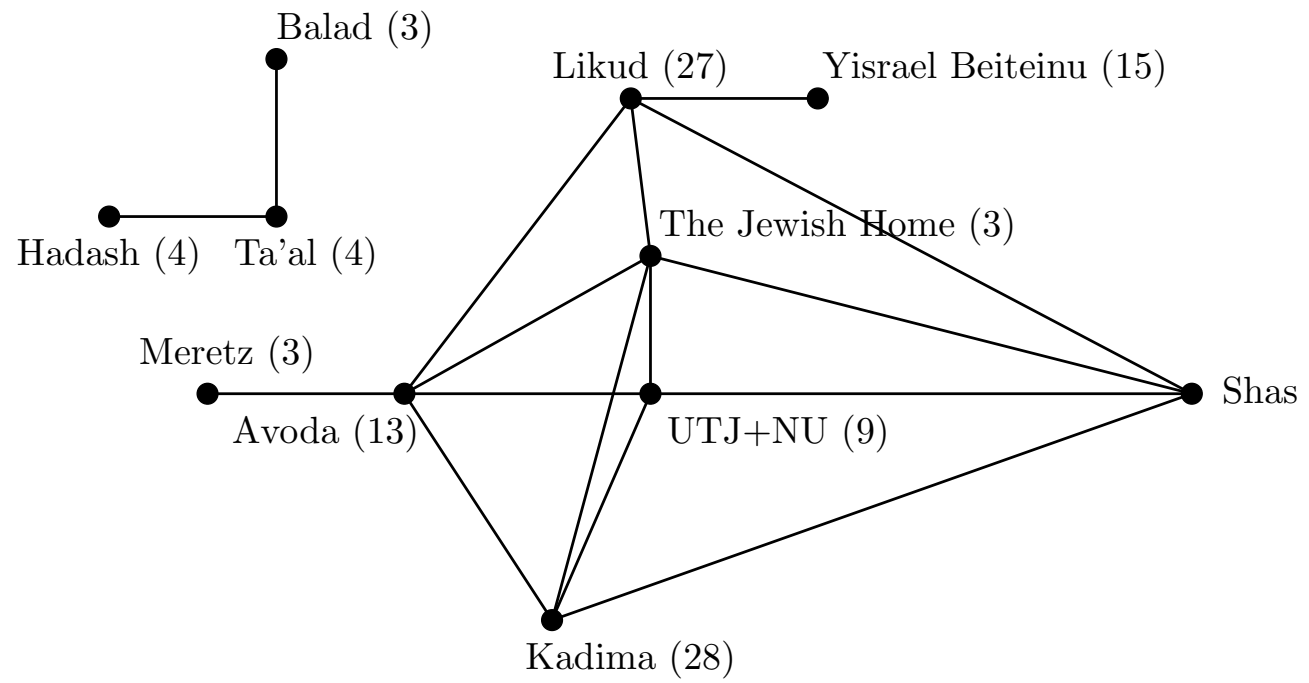

Figure 3: Israeli parliament elections 2009, network structure.

The computation results on application of the Deegan-Packel type indices are presented in Table 4.

\begin{tabular}{c|c|c|c|c|c}
\hline Party & Seats & $D P$ & $D P^{w}$ & $D P^{r}$ & $D P^{r w}$ \\
\hline \hline Kadima & 28 & 0.108 & 0.241 & 0.102 & 0.144 \\
\hline Likud & 27 & 0.106 & 0.233 & 0.224 & 0.375 \\
\hline Yisrael Beiteinu & 15 & 0.102 & 0.131 & 0.106 & 0.106 \\
\hline Avoda & 13 & 0.097 & 0.110 & 0.187 & 0.159 \\
\hline Shas & 11 & 0.085 & 0.085 & 0.165 & 0.116 \\
\hline United Torah Judaism + National Union & 9 & 0.073 & 0.063 & 0.128 & 0.080 \\
\hline Ta'al & 4 & 0.090 & 0.034 & 0 & 0 \\
\hline Hadash & 4 & 0.090 & 0.034 & 0 & 0 \\
\hline Balad & 3 & 0.083 & 0.024 & 0 & 0 \\
\hline The Jewish Home & 3 & 0.083 & 0.024 & 0.044 & 0.011 \\
\hline Meretz & 3 & 0.083 & 0.024 & 0.044 & 0.011 \\
\hline
\end{tabular}

Table 4: Israeli parliament elections 2009, Deegan-Packel like power share

The results obtained obviously show that both, the restricted Deegan-Packel and weighted restricted Deegan-Packel indices, clearly award the primacy to Likud emphasizing the advantage of its strategic position in the network.

\section{References}

[1] Banzhaf, J. (1965), Weighted voting doesn't work: a mathematical analysis, Rutgers Law Review, 19, 317-343.

[2] Brams, S.J. and Fishburn (1995), When is size a liability? Bargaining power in minimal winning coalitions, Journal of Theoretical Politics, 7, 301-316. 
[3] Brams, S.J. and Fishburn (1996), Minimal winning coalitions in weighted-majority voting games, Social Choice and Welfare, 13, 397-417.

[4] Deegan, J. and E.W. Packel (1978), A new index of power for simple $n$-person games, International Journal of Game Theory, 7, 113-123.

[5] Harsanyi, J.C. (1959), A bargaining model for cooperative $n$-person games, in: Tucker AW, Luce RD (eds.) Contributions to the theory of games IV, Princeton University Press, Princeton, NJ, pp. 325-355.

[6] Haeringer, G. (1999), Weighted Myerson value, International Game Theory Review, 1, 187-192.

[7] Kalai, E. and D. Samet (1987), On weighted Shapley values, International Journal of Game Theory, 16, 205-222.

[8] Myerson, R.B. (1977), Graphs and cooperation in games, Mathematics of Operations Research, 2, 225-229.

[9] Owen, G. (1968), A note on the Shapley value, Management Science, 14 (11), 731-732.

[10] Riker, W.H. (1962), The Theory of Political Coalitions, Yale University Press, New Haven, CT.

[11] Shapley, L.S. (1953), A value for $n$-person games, in: Tucker AW, Kuhn HW (eds.) Contributions to the theory of games II, Princeton University Press, Princeton, NJ, pp. 307-317.

[12] Shapley, L.S. and M. Shubik (1954), A method for evaluating the distribution of power in a committee system, The American Political Science Review 48, 787-792. 